\title{
Remarks on the Symmetries of Planar Fronts
}

\author{
F. AICARDI
}

\begin{abstract}
A front is the projection on the plane of a legendrian immersion of a circle in the space of the contact elements of that plane. I analyse the symmetries of a generic front with respect to the group generated by the involutions reversing the orientation of the plane, the orientation of the preimage circle and the coorientation of the contact plane.
\end{abstract}

\section{GENERIC FRONTS}

A planar front is the projection to $\mathbf{R}^{2}$ (with coordinates $x, y$ ) of a legendrian curve. A legendrian curve is the image of a $C^{1}$-immersion of $S^{1}$ in the space $M^{3}$ (with coordinates $x, y, \phi(\bmod 2 \pi)$ for cooriented fronts, $(\bmod \pi)$ for noncooriented fronts) of the contact elements of the plane, with its natural contact structure

$$
(\cos \phi) d x+(\sin \phi) d y=0
$$

We call such an immersion of $S^{1}$ into $M^{3}$ an $L$-immersion

1991 Mathematics Subject Classification: 53A04, 57M25, 58F05.

Servicio publicaciones Univ. Complutense. Madrid, 1995. 
The front is cooriented, if the contact element is cooriented, i.e. if a choice of one of two halfplanes into which it divides the tangent plane is made. We shall consider now the cooriented fronts, and $M^{3}$ will be the space of the cooriented contact elements.

The front is oriented if the preimage circle $S^{1}$ is oriented.

A generic front may have as singularities only the ordinary double points and the semicubical cusp points.

Examples. See fig.1.

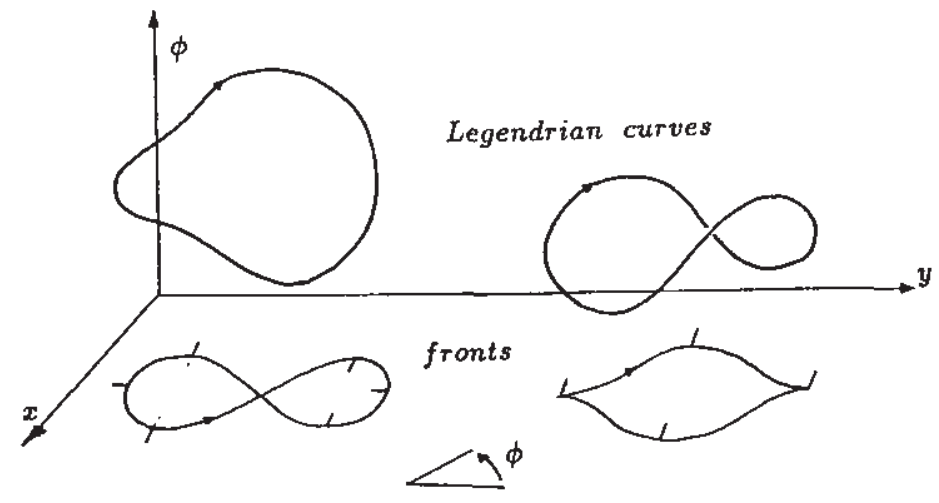

Fig. 1

Oriented legendrian curves and their oriented and cooriented fronts

To any oriented legendrian curve in the space of cooriented contact elements of the plane one associates two integer numbers: the index and the Maslov index (see [1]). Both these indices can be calculated in terms of the front of the legendrian curve.

Theorem 1. [1] The index $i$ of an oriented legendrian curve in the space $M^{3}$ is equal to the total angle (divided by $2 \pi$ ) of the rotation of the coorienting normal vector of its front when the point of the front makes a full turn along it. 
The Maslov index $\mu$ of a generic oriented legendrian curve is equal to the difference between the number $\mu^{+}$of positive cusps and the number $\mu^{-}$of negative cusps of its front: $\mu=\mu^{+}-\mu^{-}$.

A cusp of an oriented and cooriented front is called positive if the coorienting normal vector at the cusp point belongs to the halfplane bounded by the tangent line at the cusp and containing the cusp branch with the orientation going away from the cusp point.

Remarks (important) 1) The sign of the angle of rotation of the normal vector does not depend on the front coorientation, but depends on the orientation of the plane. Hence the sign of the index does not depend on the front coorientation and changes when the orientations of the plane or of the front is changed.

2) The sign of a cusp changes when one changes either the orientation or the coorientation of the front, but does not depend on the orientation of the plane. Hence the Maslov index changes its sign when the orientation or the coorientation of the front is changed and it is independent of the orientation of the plane.

Examples. See fig. 2

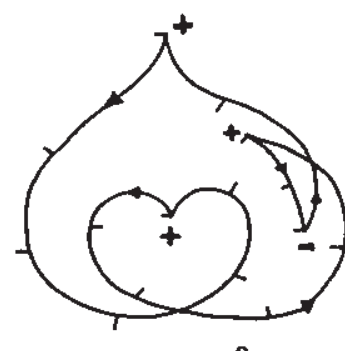

$i=2 \mu=2$

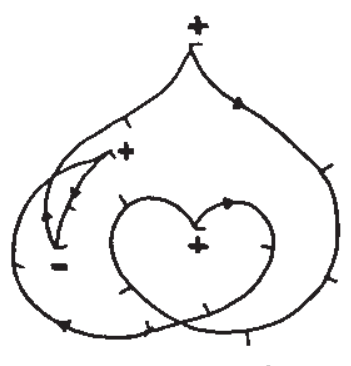

$i=-2 \quad \mu=2$

Fig. 2

Two fronts with their indices $i$ and $\mu$. 


\section{FRONT CLASSES AND SYMMETRY CLASSES}

A front class is a class of $L$-immersions having generic fronts up to orientations preserving diffeomorphisms preserving the orientations of the plane and of the preimage circle.

Consider a group $G$ acting on the space of $L$-immersions.

Definition 1. A front class $[\gamma]$ is called $G$-invariant if $[G \gamma]=[\gamma]$

Example. Let $G_{3}$ be the group of the euclideans motions in the plane. Any front class is $G_{3}$-invariant.

Let $T$ ( $T$ from Turn) ( $\left.T=S^{1} \times S^{1}\right)$ the group of the rotations of the plane and of the rotations of the preimage circle. $T$

Remark. Every front class is invariant with respect to the group

Theorem 2. If the front class $[\gamma]$ is invariant with respect to the symmetry group $H$, then there exist a front $\gamma_{*} \in[\gamma]$ so that $H \gamma_{*} \in T \gamma_{*}$, i.e. for any element $h$ of $H$ there exist an element $\tau$ in $T$ so that

$$
h \quad \gamma_{*}=\tau \quad \gamma_{*} .
$$

This theorem is stated in [2] with no proof for some special finite symmetry groups $H$.

We first consider the classification of oriented and cooriented fronts in the oriented plane up to the diffeomorphisms of the plane and of the preimage circle preserving the orientations. The front being cooriented, these diffeomorphisms preserve also the coorientation.

Let $\Omega_{i, \mu}$ be the space of immersions of the oriented circle into the space of the cooriented contact elements of the oriented plane with fixed index $i$ and Maslov index $\mu$, and $\mathcal{N}_{|i|,|\mu|}$ the corresponding space if only the absolute values of the indices are fixed. In this second space, consider the action of the following involutions:

Involution $\Sigma$ : it reverses the orientation of the plane.

Involution $\sigma$ : it reverses the orientation of the preimage circle. 
Involution $\Theta$ : it reverses the coorientation of the front.

We represent an element of $\mathcal{N}_{|i|,|\mu|}$ as the graph of the application of the circle into $M^{3}$, i.e. as a curve $\bar{\gamma}$ living in the space $S^{1} \times M^{3}$ with coordinates

$$
t(\bmod 2 \pi), \quad x(t), \quad y(t), \quad \phi(t)(\bmod 2 \pi)
$$

The action of an element $\tau_{(\alpha, \theta)}$ of $T$ on an $L$-immersion, i.e. the rotation of the preimage circle of an angle $\alpha$ and the rotation of an angle $\theta$ in the plane $(x, y)$ is thus represented by the following transformation of $\tilde{\gamma}$ into $\tilde{\gamma}^{\prime}$ :

$$
\begin{aligned}
t^{\prime} & =t+\alpha \\
x^{\prime} & =(\cos \theta) x-(\sin \theta) y \\
y^{\prime} & =(\cos \theta) y+(\sin \theta) x \\
\phi^{\prime} & =\phi+\theta
\end{aligned}
$$

Moreover the involutions $\Sigma, \sigma$ and $\Theta$ acting on the front classes can be represented with the following matrices acting on the immersions respectively:

involution $\Sigma$ :

$$
a=\left(\begin{array}{cccc}
1 & 0 & 0 & 0 \\
0 & -1 & 0 & 0 \\
0 & 0 & 1 & 0 \\
0 & 0 & 0 & -1
\end{array}\right)
$$

involution $\sigma$ :

$$
b=\left(\begin{array}{cccc}
-1 & 0 & 0 & 0 \\
0 & 1 & 0 & 0 \\
0 & 0 & 1 & 0 \\
0 & 0 & 0 & 1
\end{array}\right)
$$

involution $\Theta$ :

$$
c=\left(\begin{array}{cccc}
1 & 0 & 0 & 0 \\
0 & -1 & 0 & 0 \\
0 & 0 & -1 & 0 \\
0 & 0 & 0 & 1
\end{array}\right)
$$


These involutions generate a commutative group $R O$ (Reversing Orientations) of order 8 , whose elements coincide with their inverse ones. They are denoted by

$$
I, \quad a, \quad b, \quad c, \quad a b, \quad a c, \quad b c, \quad a b c .
$$

Let $H$ be the symmetry group $R O$ or a subgroup of $R O$. We want find the classes invariant under the action of the symmetry group $H$. Hence, using theorem 2, we search for a solution $\left(\tilde{\gamma}_{H}, \tau_{i}\right)$ of the system of equations

$$
h_{i} \bar{\gamma}_{H}=\tau_{i} \bar{\gamma}_{H}
$$

where $h_{i}$ are the generators of the group $H$ and $\tau_{i}$ are particular elements of the group $T$. If such a solution exists, then the front class containing $\gamma_{H}$ will be the requested $H$-invariant class.

(1)

Fig. 3

The eight asymmetric classes 
Definition 2. We call asymmetric class a front class which is not invariant by any subgroup of $R O$ different from the identity.

The action of $R O$ on an asymmetric class of $\mathcal{N}_{|i|,|\mu|}$ transforms it into 8 different asymmetric classes, with different values of the signs of the nonzero indices.

Example. See fig. 3 .

Definition 3. We call simply symmetric class a front class which is invariant only with respect to a subgroup of order two of $R O$.

Every element $\rho$ of $R O$ different from the identity $I$ generates a subgroup of $R O$ of order 2 . We denote by $R_{(\rho)}$ this subgroup, by $\left[\gamma_{\rho}\right]$ the class invariant only with respect to $R_{(\rho)}$, by $\tilde{\gamma}_{\rho}$ the curve, solution of equation (7), and by $\gamma_{\rho}$ its corresponding front. The action of $R O$ on a simply symmetric class transform it into 4 different simply symmetric classes in $\mathcal{N}_{|\hat{i}|,|\mu|}$.

Definition 4. We call supersymmetric class a front class which is invariant with respect to a subgroup of order four of $R O$.

Two elements $\rho_{1}$ and $\rho_{2}$ of $R O$ different from the identity $I$ generate a subgroup of $R O$ of order $4\left(I, \rho_{1}, \rho_{2}, \rho_{1} \rho_{2}\right)$. We denote by $R_{\left(\rho_{1}\right)\left(\rho_{2}\right)}$ this subgroup, by $\left[\gamma_{\rho_{1}, \rho_{2}}\right]$ its invariant class, by $\tilde{\gamma}_{\rho_{1}, \rho_{2}}$ the curve, solution of equation (7) and by $\gamma_{\rho_{1}, \rho_{2}}$ its corresponding front. The action of $R O$ on this class transforms it into 2 different supersymmetric classes in $\mathcal{N}_{|i|,|\mu|}$

Theorem 3. i) There are no invariant classes under the action of the entire group $R O$. ii) The front classes invariant with respect to the subgroups $R_{(a)}, R_{(c)}, R_{(a b)}, R_{(a c)}, R_{(b c)}, R_{(a b c)}, R_{(a)(b c)}, R_{(c)(a b)}$, $R_{(a b)(b c)}$ are not void. They are listed in the following table, where the solutions of eq. (7) for every subgroup are shown in the identities in the second column. 


\begin{tabular}{|c|c|c|c|c|}
\hline Subgroup & Identities & $\begin{array}{l}\text { Number } \\
\text { of inv. cl. }\end{array}$ & $\begin{array}{l}\text { Space } \\
\text { of inv. cl. }\end{array}$ & $\begin{array}{l}\text { Examples } \\
\text { in figg. }\end{array}$ \\
\hline$R_{(a)}=(I, a)$ & $a \tilde{\gamma}_{a}=\tau_{\pi, 0} \bar{\gamma}_{a}$ & 4 & $\mathcal{N}_{0,|\mu|}$ & 4,5 \\
\hline$R_{(\mathrm{c})}=(I, c)$ & $c \tilde{\gamma}_{c}=\tau_{\pi+\alpha, \alpha} \tilde{\gamma}_{c}\left(^{*}\right)$ & 4 & $\mathcal{N}_{|i|, 0}$ & 6,7 \\
\hline$R_{(a b)}=(I, a b)$ & $a b \bar{\gamma}_{a b}=\tau_{0,0} \bar{\gamma}_{a b}$ & 4 & $\mathcal{N}_{|i|, 0}$ & 8,9 \\
\hline$R_{(a c)}=(I, a c)$ & $a c \bar{\gamma}_{a c}=\tau_{\pi, 0} \tilde{\gamma}_{a c}$ & 4 & $\mathcal{N}_{0,0}$ & 10,11 \\
\hline$R_{(b c)}=(I, b c)$ & $b c \tilde{\gamma}_{b c}=\tau_{0,0} \tilde{\gamma}_{b c}$ & 4 & $\mathcal{N}_{0,|\mu|}$ & 12,13 \\
\hline$R_{(a b c)}=(I, a b c)$ & $a b c \tilde{\gamma}_{a b c}=\tau_{0,0} \quad \tilde{\gamma}_{a b c}$ & 4 & $\mathcal{N}_{|i|,|\mu|}$ & 14,15 \\
\hline $\begin{array}{c}R_{(a),(b c)}= \\
(I, a, b c, a b c)\end{array}$ & $\begin{array}{l}a \tilde{\gamma}_{a, b c}=\tau_{\pi, 0} \tilde{\gamma}_{a, b c} \\
b c \tilde{\gamma}_{a, b c}=\tau_{0,0} \quad \tilde{\gamma}_{a, b c}\end{array}$ & 2 & $\mathcal{N}_{0,|\mu|}$ & 16,17 \\
\hline $\begin{array}{c}R_{(c),(a b)}= \\
(I, c, a b, a b c)\end{array}$ & $\begin{array}{c}c \bar{\gamma}_{c, a b}=\tau_{\pi+\alpha, \alpha} \tilde{\gamma}_{c, a b}\left({ }^{*}\right) \\
a b \tilde{\gamma}_{c, a b}=\tau_{0,0} \tilde{\gamma}_{c, a b}\end{array}$ & 2 & $\mathcal{N}_{|i|, 0}$ & 18,19 \\
\hline $\begin{array}{c}R_{(a b)(b c)}= \\
(I, a b, b c, a c)\end{array}$ & $\begin{array}{l}a b \tilde{\gamma}_{a b, b c}=\tau_{\pi, 0} \tilde{\gamma}_{a b, b c} \\
b c \tilde{\gamma}_{a b, b c}=\tau_{0,0} \tilde{\gamma}_{a b, b c}\end{array}$ & 2 & $\mathcal{N}_{0,0}$ & 20,21 \\
\hline
\end{tabular}

Proof. Consider the subgroup $R_{(b)}=(I, b)$. The solution of the equation (7) for the symmetric front $\gamma_{b}$ does not exist. According to theorem 2 , there are not $R_{(b)}$-invariant front classes. It follows that also the group $R O$ cannot have invariant classes, because they would be symmetric with respect to this subgroup $R_{(b)}$.

The other subgroups of $R O$ of order two have invariant classes. Indeed, according to theorem 2, the equation (7) has solutions in the forms shown in the table. Such solutions are unique, in the sense that 
in every simply symmetric class $\left[\gamma_{\rho}\right]$ there exist an element $\gamma_{\rho}$ which satisfies the corresponding identity shown in the second column. The simply symmetric fronts explicitly satisfy respectively:

the front $\gamma_{a}$ satisfies:

$$
\begin{aligned}
-x(t) & =x(t+\pi) \\
y(t) & =y(t+\pi) \\
-\phi(t) & =\phi(t+\pi)
\end{aligned}
$$

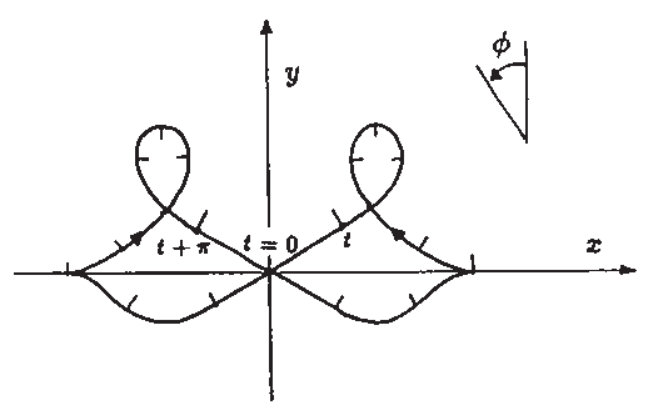

Fig.4

A simply symmetric front $\gamma_{a}$ with $i=0$ and $\mu=2$

the front $\gamma_{c}$ satisfies:

$$
\begin{aligned}
-x(t) & =(\cos \alpha) x(t+\pi+\alpha)-(\sin \alpha) y(t+\pi+\alpha) \\
-y(t) & =(\cos \alpha) y(t+\pi+\alpha)+(\sin \alpha) x(t+\pi+\alpha) \\
\phi(t) & =\phi(t+\pi+\alpha)+\alpha
\end{aligned}
$$

where $\alpha=(2 n+1) \frac{\pi}{p}$ (symmetry of order $p$ even), or $\alpha=\frac{2 n \pi}{p}$ (symmetry of order $p$ odd). An example of no rotation symmetry, i.e. the case $p=1$ $(\alpha=2 \pi n)$ is shown in fig. 6. An example of rotation symmetry $(p=2)$ is shown in fig. 22 


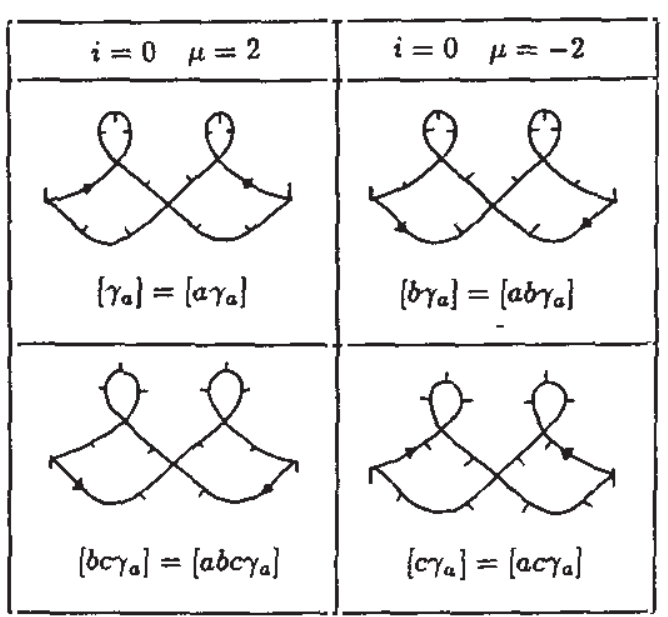

Fig.5

The four $R_{(a)}$-invariant classes in $\mathcal{N}_{0,|\mu|}$

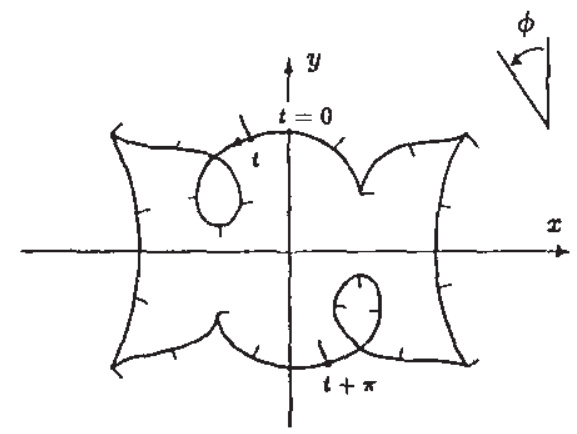

Fig.6

A simply symmetric front $\gamma_{c}$ with $i=2$ and $\mu=0$ 


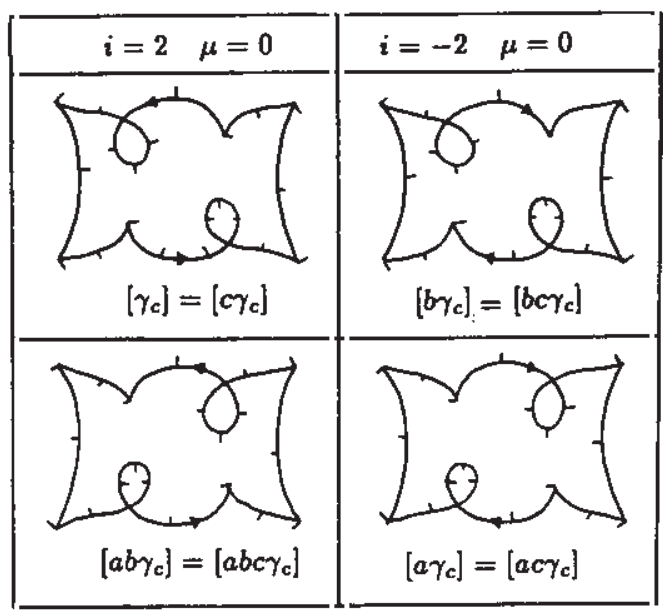

Fig.7

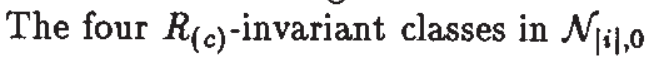
the front $\gamma_{a b b}$ satisfies:

$$
\begin{array}{r}
-x(-t)=x(t) \\
y(-t)=y(t) \\
-\phi(-t)=\phi(t)
\end{array}
$$




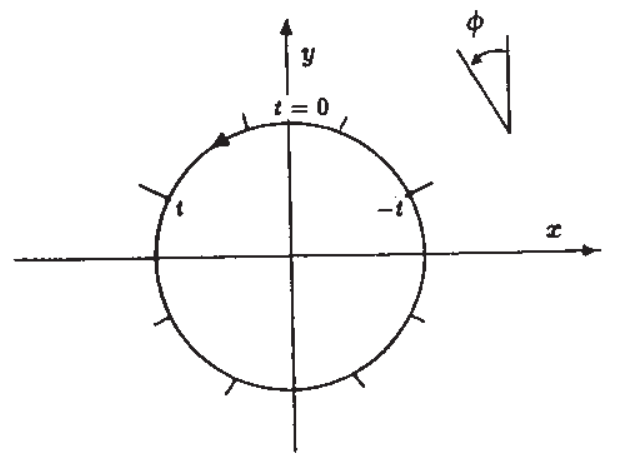

Fig.8

A simply symmetric front $\gamma_{a b}$ with $i=1$ and $\mu=0$

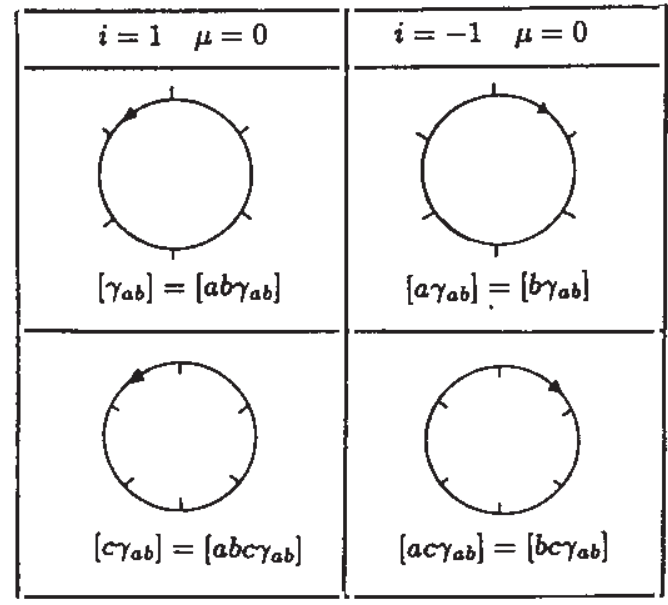

Fig.9

The four $R_{(a b)}$-invariant classes in $\mathcal{N}_{|i|, 0}$ 
the front $\gamma_{a c}$ satisfies:

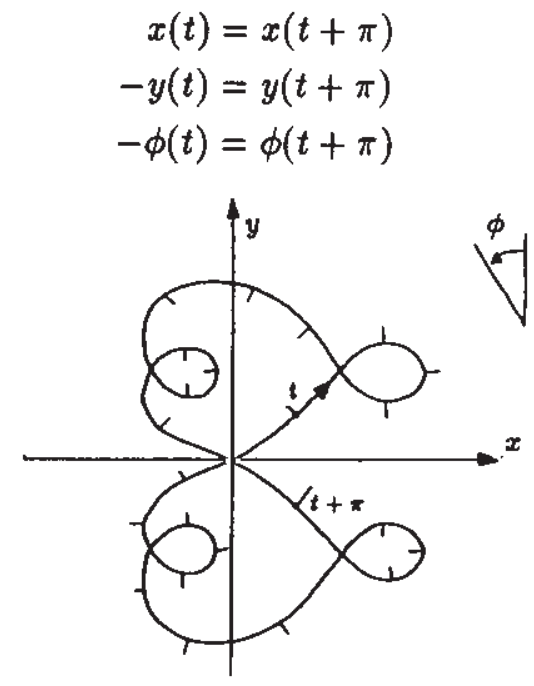

Fig.10

A simply symmetric front $\gamma_{a c}$ with $i=0$ and $\mu=0$

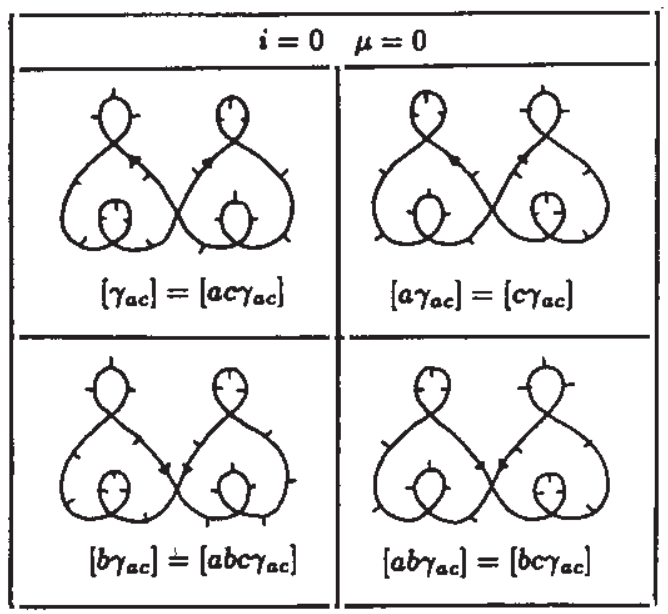

Fig.11

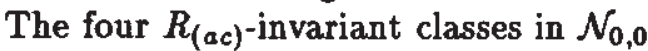


the front $\gamma_{b c}$ satisfies:

$$
\begin{array}{r}
-x(-t)=x(t) \\
-y(-t)=y(t) \\
\phi(-t)=\phi(t)
\end{array}
$$

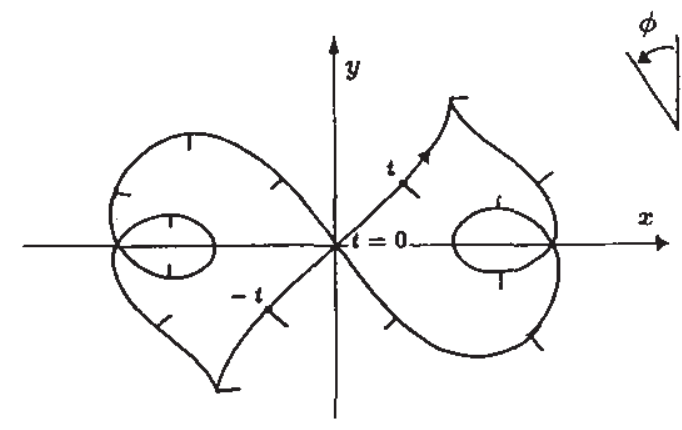

Fig.12

A simply symmetric front $\gamma_{b c}$ with $i=0$ and $\mu=2$

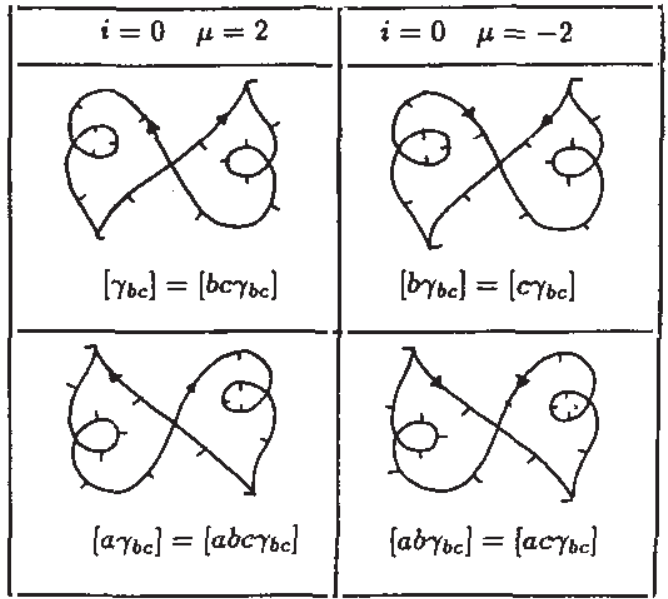

Fig.13

The four $R_{(b c)}$-invariant classes in $\mathcal{N}_{0,|\mu|}$ 
the front $\gamma_{a b c}$ satisfies:

$$
\begin{array}{r}
x(-t)=x(t) \\
-y(-t)=y(t) \\
-\phi(-t)=\phi(t)
\end{array}
$$

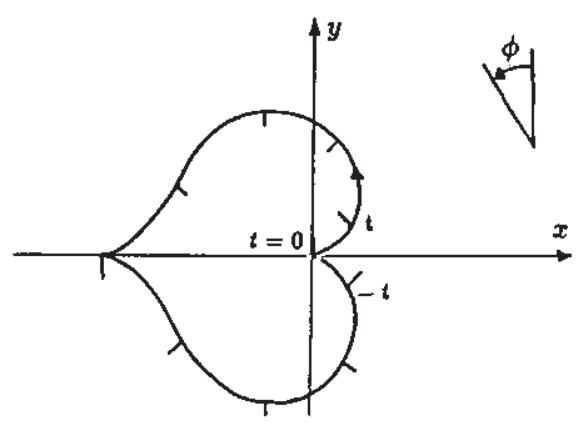

Fig.14

A simply symmetric front $\gamma_{a b c}$ with $i=1$ and $\mu=2$

in

Fig.15

The four $R_{(a b c)}$-invariant classes in $\mathcal{N}_{|i|,|\mu|}$ 
Consider now the subgroups of order four.

Subgroups $R_{(a),(b)}, \quad R_{(b),(c)}, \quad R_{(b),(a c)}$ cannot have invariant classes, because their invariant classes would also be invariant with respect to $R_{(b)}$, which does not have invariant classes.

The subgroup $R_{(a),(c)}=(I, \quad a, \quad c, a c)$ has no invariant classes. The equation (7) has no solution, indeed the supersymmetric front would satisfy both (8) and (11), which are incompatible.

The other subgroups of $R O$ of order 4 have invariant classes. The solutions of eq. (7) are shown in the table, i.e. the supersymmetric fronts satisfy respectively:

the front $\gamma_{a, b c}$ satisfies:

$$
\begin{aligned}
-x(t) & =x(t+\pi) \\
y(t) & =y(t+\pi) \\
-\phi(t) & =\phi(t+\pi)
\end{aligned}
$$

and

$$
\begin{aligned}
-x(-t) & =x(t) \\
-y(-t) & =y(t) \\
\phi(-t) & =\phi(t)
\end{aligned}
$$

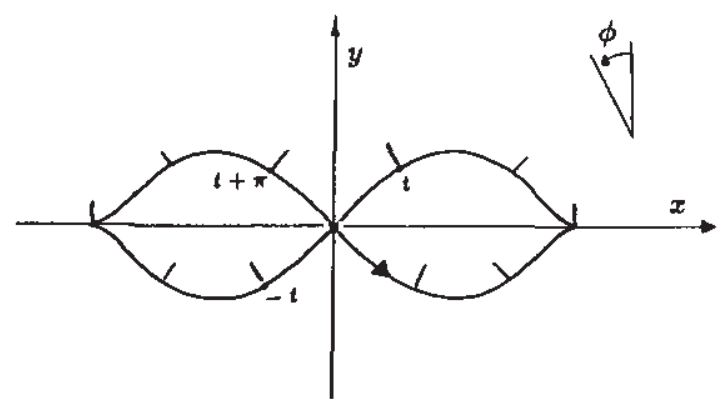

Fig.16

A supersymmetric front $\gamma_{a, b c}$ with $i=0$ and $\mu=2$. 


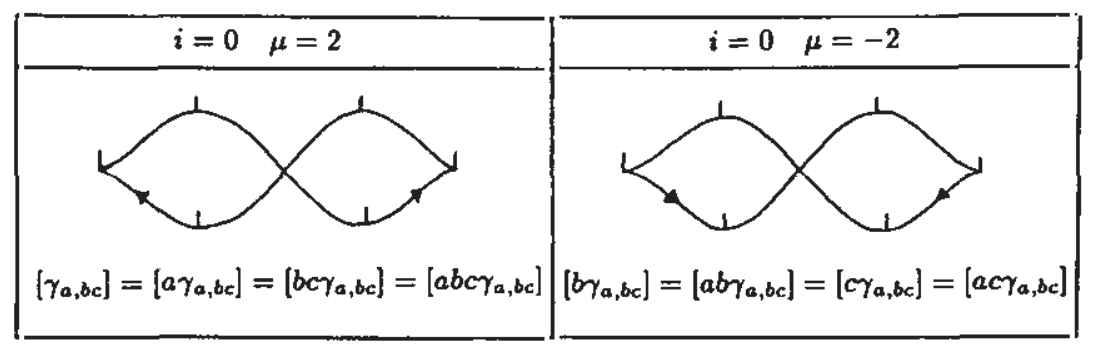

\section{Fig.17}

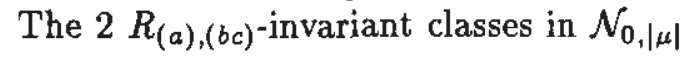

the front $\gamma_{c, a b}$ satisfies:

$$
\begin{aligned}
-x(t) & =(\cos \alpha) x(t+\pi+\alpha)-(\sin \alpha) y(t+\pi+\alpha) \\
-y(t) & =(\cos \alpha) y(t+\pi+\alpha)+(\sin \alpha) x(t+\pi+\alpha) \\
\phi(t) & =\phi(t+\pi+\alpha)+\alpha
\end{aligned}
$$

where $\alpha=(2 n+1) \frac{\pi}{p}$ (symmetry of order $p$ even), or $\alpha=\frac{2 n \pi}{p}$ (symmetry of order $p$ odd).

and

$$
\begin{array}{r}
-x(-t)=x(t) \\
y(-t)=y(t) \\
-\phi(-t)=\phi(t)
\end{array}
$$


In fig. 18 examples of the case of no rotation symmetry $(p=1)$ and of rotation symmetry $(p=2)$ are given. An example of the case $p=3$ is shown in fig. 22.

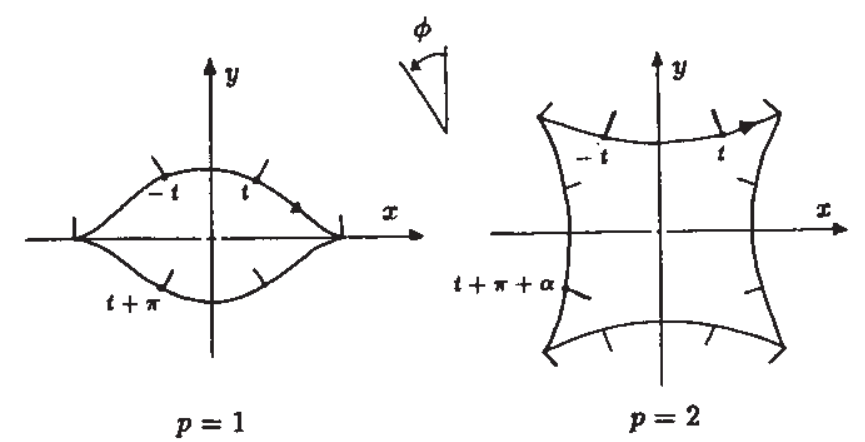

Fig.18

Supersymmetric fronts $\gamma_{c, a b}$ with $\mu=0, i=0$ and $\mu=0, i=1$

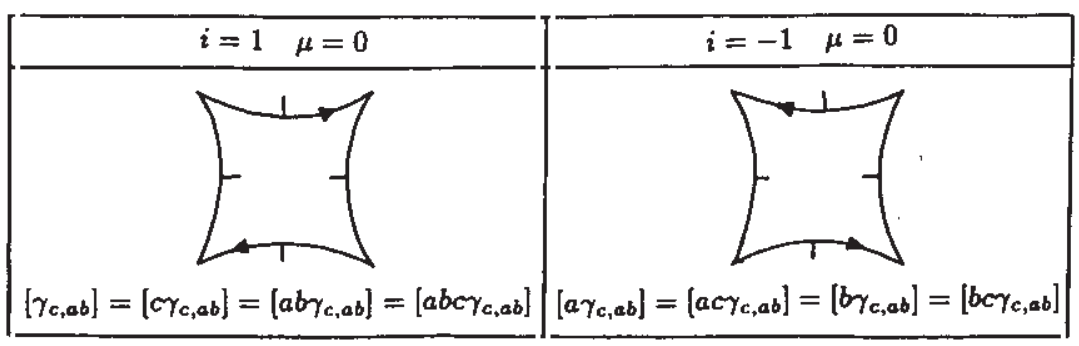

Fig.19

The $2 R_{(c),(a b)}$-invariant classes in $\mathcal{N}_{|i|, 0}$ 
the front $\gamma_{a b, b c}$ satisfies:

$$
\begin{aligned}
-x(-t) & =x(t+\pi) \\
y(-t) & =y(t+\pi) \\
-\phi(-t) & =\phi(t+\pi)
\end{aligned}
$$

and

$$
\begin{array}{r}
-x(-t)=x(t) \\
-y(-t)=y(t) \\
\phi(-t)=\phi(t)
\end{array}
$$

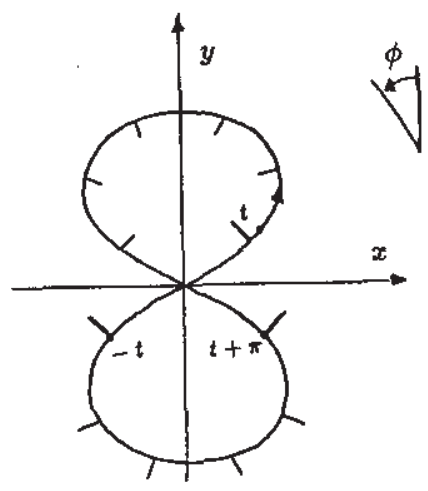

Fig.20

A supersymmetric front $\gamma_{a b, b c}$ with $i=0$ and $\mu=0$

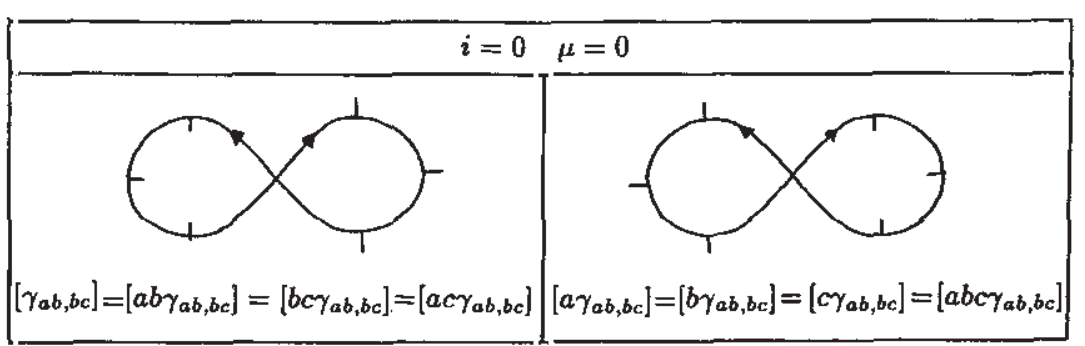

Fig.21

The $2 R_{(a b),(b c)}$-invariant classes in $\Omega_{0,0}$ 


\section{ROTATION SYMMETRIES}

Definition. A planar front $\gamma$ is called p-symmetric if

$$
\tilde{\gamma}=\tau_{\alpha, \rho} \tilde{\gamma}
$$

where $\beta=\frac{2 \pi}{p}, \alpha= \pm \beta$

Proposition. Every p-symmetric front $(p>1)$ belongs to one of the \& following symmetry classes:

$$
\left[\gamma_{a b}\right], \quad\left[\gamma_{a b c}\right], \quad\left[\gamma_{c}\right], \quad\left[\gamma_{c, a b}\right]
$$

Proof. The index $i$ of a $p$-symmetric front is $i=p \times i_{p}$ where $2 \pi i_{p}$ is the total rotation angle of the normal vector along a $p$-th part of the front (for example in the interval $[t, t+\beta]$ of the preimage circle). By hypothesis $\phi(t)=\phi(t+\beta) \pm \beta$ (modulo $2 \pi)$ so that $2 \pi i_{p}=\phi(t+\beta)-\phi(t)$ cannot be zero.

Thus the symmetries allowed are those with index different from zero. For every type of such symmetries we give examples in fig. 22 .

\section{OTHER TYPES OF CLASSIFICATION}

The above classification contains less refined classifications.

For example, we can consider the classification problem when the front class is taken up to diffeomorphisms not necessarily preserving the orientation of the circle. The answer is thus given by the above table, where the element $b$ is everywere substituted by the identity. The quotient group $(I, a, c, a c)$ has in this case one invariant class, corresponding of course to the supersymmetric class $\left[\gamma_{c, a b}\right]=\left[\gamma_{a b, b c}\right]$

Remark. In all such less refined classifications, all subgroups have invariant classes. 

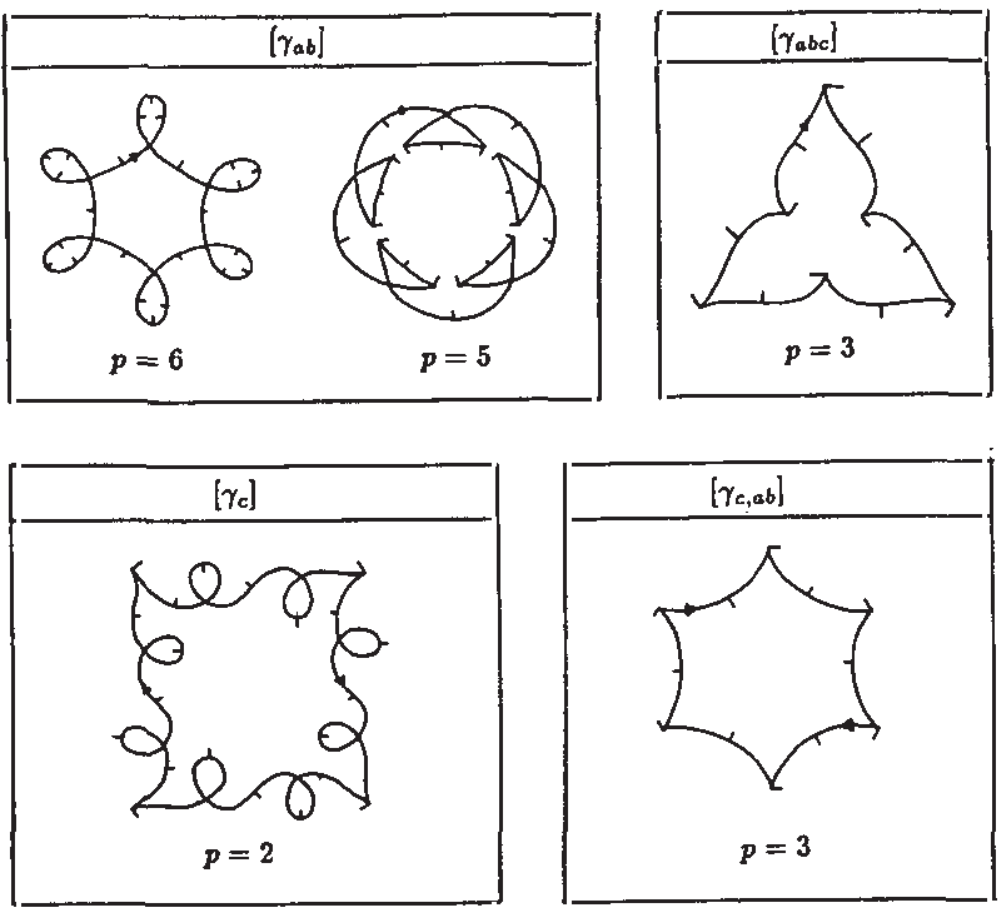

Fig.22

$p$-symmetric fronts with their $R O$-symmetries

Example. The involution $\sigma$ considered in $[1,2]$ acting on the $C^{1}-$ immersions of the circle into the plane (the coorientation defined by the normal vector being not considered) has invariant classes.

The classification "up to the change of an orientation" can be also seen as a classification forgetting such orientation. Hence the quotient group $(I, a, b, a b)$ acts also on planar nonorientable fronts with semiinteger index (i.e. with an odd number of cusps), which can be defined as projections onto the plane of legendrian immersions of $S^{1}$ into the space of non oriented contact elements of the plane $\hat{M}^{3}=P T^{*} \mathrm{R}^{2}$ (where $M^{3} \rightarrow \hat{M}^{3}$ is the double covering.) 


\section{DIVERTISSEMENT}

Associate to every smooth segment of a oriented and cooriented front in the oriented plane a sign $s$ in the following way: if the pair (orienting tangent vector, coorienting normal vector) defines the positive orientation of the plane $(x, y)$, then $s=1$, otherwise $s=-1$.

The sign changes at every cusp of the front.

Define now the length $l$ of a front as the algebraic sum of the lengths of the pieces with their signs. We have associated to the front a real number $l$ (its length).

Remark. The sign of $l$, if there are cusps, is not an invariant of the front class (see fig.23)

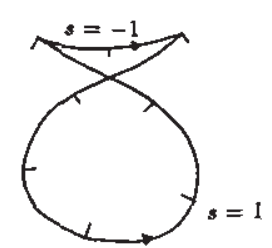

$l>0$

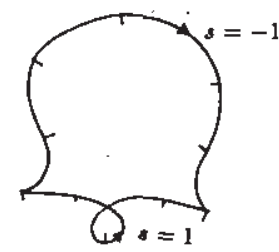

$l<0$

Fig. 23

2 fronts of the same front class and opposite values of the sign of $l$

However $l$ is invariant in every $T$-class. The analysis of symmetries, by theorem 2, is made inside $T$-classes, so that we use the number $l$ as an invariant.

The sign $l$ of a front changes for all the involutions $\Sigma, \sigma$ and $\Theta$. This means that the fronts which are invariant with respect to the subgroups $R_{(a)}, R_{(b)}, R_{(c)}$ and $R_{(a b c)}$ have all length zero, and those which are invariant for the other ones can have positive or negative lengths.

We visualize the symmetries of the group $R O$ acting on the fronts using a cube (see fig. 24): the 8 vertices of the cube represent an asymmetric front with index $i$, Maslov index $\mu$ and length $l$ under the action of the 8 elements of $R O$. 


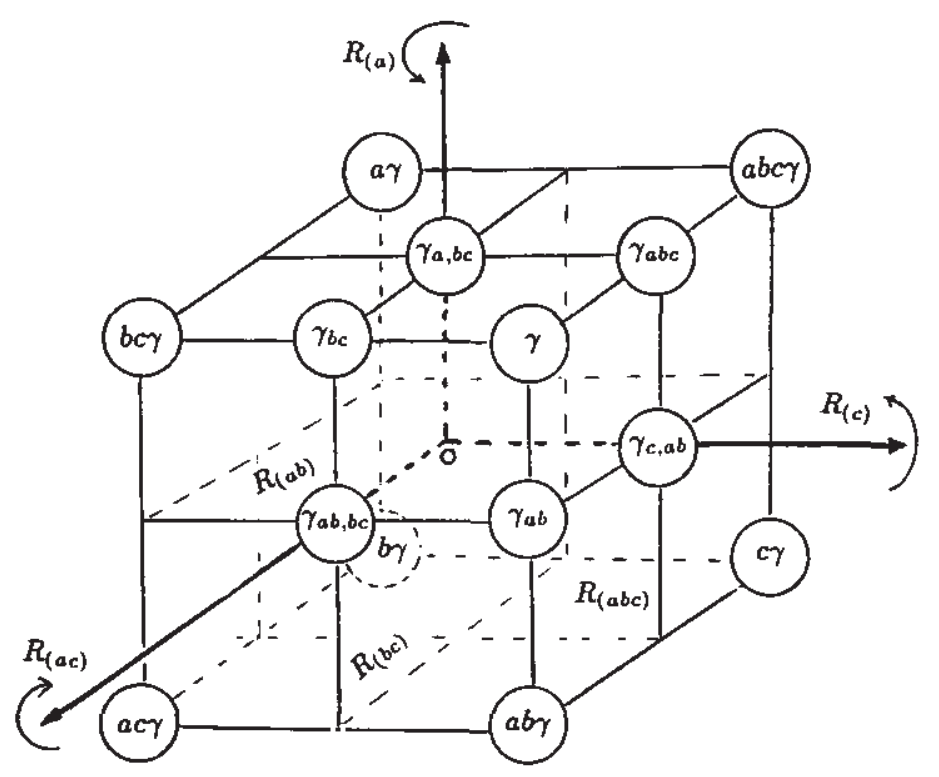

Fig. 24

The cube of the symmetries $R O$

The cube belongs to 3 -dimensional space with coordinates $(l, \mu, i)$ so that different values correspond to every vertex.

The planes $l=0, \mu=0$ and $i=0$ represent the fronts invariant under the action of the subgroups of order two $R_{(a b c)}, R_{(a b)}$ and $R_{(b c)}$ respectively (the action is the reflection on these planes).

The rotations by an angle $\pi$ around the 3 principal axes represent the other 3 subgroups of order two: $R_{(a)}(i=0, l=0), R_{(c)}(\mu=0, l=$ $0)$, and $R_{(a c)}(i=0, \mu=0)$.

The principal axes represent the fronts invariant under the action of the subgroups of order four. Every axis is in fact invariant under the 
rotation around itself and under the reflection with respect to the planes containing it.

Remark. In this scheme the subgroups $R_{(b)}$ and $R_{(a, c)}$, which have no invariant classes, leave invariant only the origin.

\section{References}

[1] V.I.Arnold Invarianty $i$ perestroiki ploskikh frontov, Trudy Mian, vol. 209, 1995.

[2] V.I.Arnold Plane Curves, Their Invariants, Perestraikas and Classifications, Adv. in Sov. Math., A.M.S. 21, Providence, 1994.

International Centre for Theoretical Physics

Recibido: 4 de Agosto de 1994

Strada Costiera, 11

I-34014 Trieste

ITALIA 